\title{
Photocatalytic Hydrogen Generation Coupled to Pollutant Utilisation Using Carbon Dots Produced from Biomass
}

Demetra S. Achilleos ${ }^{a, \dagger}$, Hatice Kasap ${ }^{a}$ and Erwin Reisnera,*

aChristian Doppler Laboratory for Sustainable SynGas Chemistry, Department of Chemistry, University of Cambridge, Lensfield Road, Cambridge CB2 1EW (UK) † Present Address: School of Chemistry, University College Dublin, Science Center South, Belfield, Dublin 4.

*Corresponding author: reisner@ch.cam.ac.uk 


\begin{abstract}
Photocatalysis is deemed as an appealing strategy to exploit solar energy for simultaneous fuel production and pollutant utilisation. However, current photocatalytic systems rarely couple both processes and suffer from restricted scalability and sustainability as they use toxic and/or ultraviolet light harvesters, combined with noblemetal co-catalysts under corrosive conditions. Here, we show the synthesis of ultrascalable and low-cost carbon nanodots from lignocellulosic waste, which when combined with a non-precious Ni-based co-catalyst, use visible light to drive $\mathrm{H}_{2}$ production in untreated river and sea water. Organic pollutants and chloride anions in these untreated media do not only allow unhindered photocatalytic activities, but also function as electron donors leading to economical pollutant utilisation. This system combines Earth's most abundant resources (biomass, solar energy, untreated water), and functions at ambient temperature, pressure and physiological $\mathrm{pH}$ creating perspectives for simultaneous fuel synthesis and pollutant utilisation of sustainable and practical character.
\end{abstract}




\section{Introduction}

Solar-driven catalysis has the potential to store the abundant energy of sunlight in high energy density fuels, such as $\mathrm{H}_{2}\left(120 \mathrm{MJ} \mathrm{kg}^{-1}\right)$, simultaneously with aqueous pollutant utilisation at ambient conditions. ${ }^{1}$ However, the implementation of environmentally and economically sustainable processes for such purposes requires scalable and robust photo-catalysts of low cost and toxicity, which operate with good performances in untreated water resources. Many photoabsorbers under intensive investigation, such as $\mathrm{TiO}_{2}$, cadmium chalcogenide quantum dots, ruthenium and organic dyes, as well as carbon nitride, suffer from limited visible-light absorption, toxicity, instability or poor interfacial interactions and electron transfer kinetics. ${ }^{2}$

Carbon dots (CDs) are ideal photocatalysts for such applications, since they overcome all these limitations, and they can be readily prepared from calcination of molecular

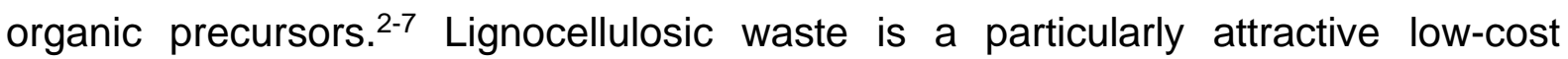
precursor for CD synthesis, as it is the largest sustainable source of organic carbon worldwide $\left(2.2 \times 10^{14} \mathrm{~kg}\right),{ }^{8,9}$ and does not compete with food production ${ }^{10}$ and biofuel synthesis. ${ }^{11}$ Biomass-derived CDs are currently under investigation in bioimaging, ${ }^{12}$ drug delivery ${ }^{13}$ and selective ion sensing, ${ }^{14-16}$ but their use in photocatalysis is less common and restricted in photoreduction of $\mathrm{Au}^{3+} /$ graphene oxide composites ${ }^{17}$ and organic oxidation without 'green' fuel formation. ${ }^{18-21}$

Most photocatalytic systems do not only rely on non-scalable and toxic photocatalysts, but also require operation in organic solvents and/or purified water, in the presence of expensive sacrificial electron donors (SEDs). ${ }^{22,} 23$ These requirements prevent currently the implementation of an economic process, particularly in areas without access to plentiful clean water. ${ }^{24,25}$ Demonstrations of practical photocatalysis using untreated river and seawater are rare,${ }^{26}$ due to the restricted durability of the catalysts, 
especially at high salinities. ${ }^{27}$ In addition, the majority of systems use expensive noblemetal co-catalysts (Pt), usually combined with UV-light $\mathrm{TiO}_{2}$ absorbers, to drive $\mathrm{H}_{2}$ production in extremely acidic synthetic water, using targeted pollutants as SEDs (Table S1). ${ }^{28-30}$ However, such studies ignore significant light attenuation effects from organic substances, which coexist with pollutants at high concentrations in untreated water, and often absorb on the photocatalyst surface. ${ }^{31}$ Latest advances in the field involve sensitisation with multiple photoabsorbers (i.e., CdS, ${ }^{32}$ carbon nitride ${ }^{33}$ ) to enhance visible light absorption (Table S1). This strategy has been traditionally applied to systems which only degrade pollutants without fuel production, relying on hydroxyl radicals generated from $\mathrm{O}_{2}$ reduction and water oxidation(Tables S2,S3). ${ }^{34}$ Photocatalysis in artificial sea water using $\mathrm{CuO} / \mathrm{TiO}_{2}{ }^{35}$ and $\mathrm{MoS} / \mathrm{TiO}_{2},{ }^{36}$ showed suppressed $\mathrm{H}_{2}$ production compared to pure water, due to $\mathrm{Cl}^{-}$adsorption on $\mathrm{TiO}_{2}$ to form $\mathrm{TiCl}$, showcasing the need for alternative materials. Thus, non-toxic, preciousmetal free and single-atom photocatalysts that are robust in untreated water and scalable at low cost, could open new avenues toward applications in the field. ${ }^{26,31,37}$ Herein, we show to overcome these limitations by utilising Earth's most abundant resources, biomass waste, sunlight and untreated water, to simultaneously produce a "green" fuel and utilise aqueous pollutants. Pure lignocellulosic biomass components, $\alpha$-cellulose, xylan and lignin, as well as crude biomass sources from leaves and cotton, were employed as precursors for the synthesis of visible-light absorbing CDs (Fig. 1a,b and Fig. S1). The biomass-derived CDs were first studied for $\mathrm{H}_{2}$ evolution in purified water (Fig. 1C) in the presence of ethylenediaminetetraacetic acid (EDTA) as SED and with a molecular bis(diphosphine) Ni co-catalyst (NiP) (Fig. 1c, S2). ${ }^{3,4}$ Under real-world conditions operating in untreated sea and river water, no external SED is 
required for this $\mathrm{CD} / \mathrm{NiP}$ system, and hole scavenging occurs by oxidising organic pollutants and anionic species present in these media (Fig. 1C).
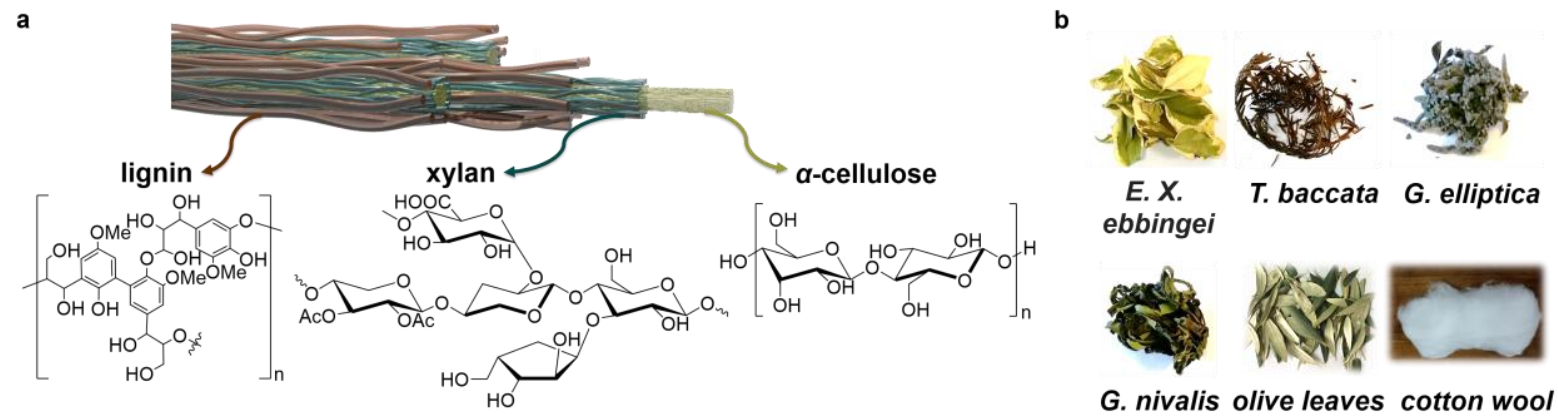

c

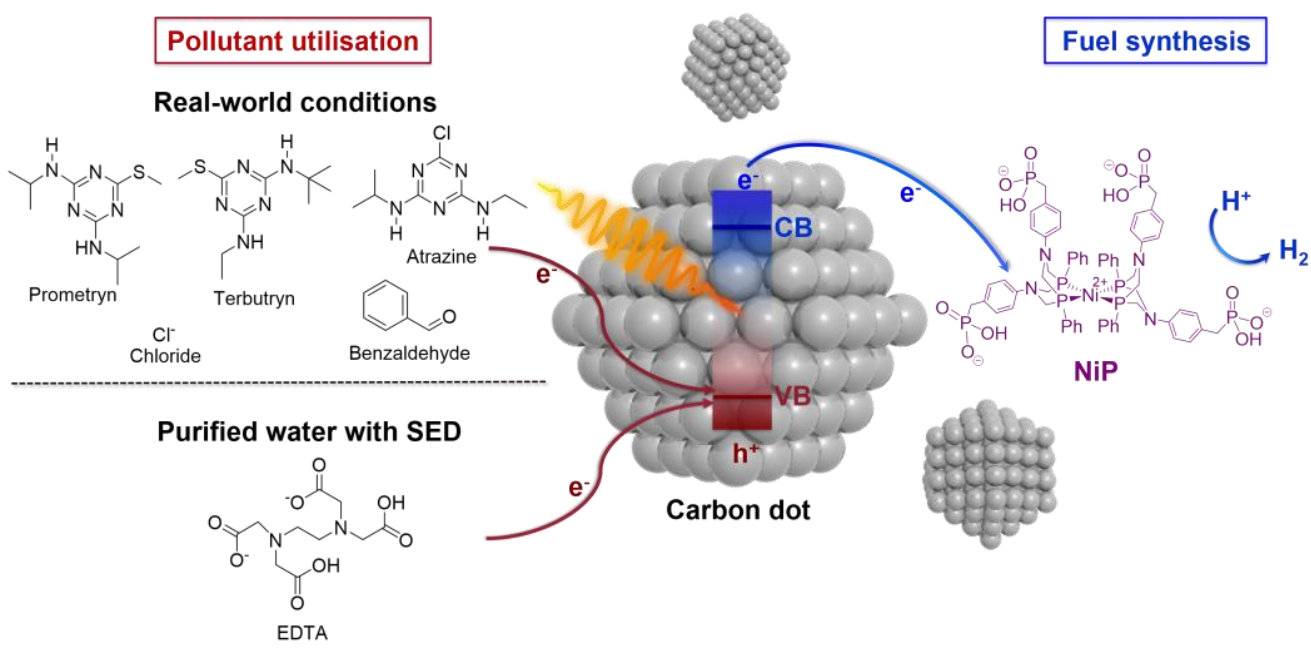

Fig. 1. a, Schematic representation of lignocellulosic structure with its lignin, xylan and $\alpha$-cellulose components used for CD synthesis. b. Optical images of locally sourced biomass waste used for the synthesis of CDs. c, Schematic representation of the photocatalytic $\mathrm{H}_{2}$ evolution and pollutant utilisation system, composed of CDs (lightharvesters) and NiP $\left(\mathrm{H}_{2}\right.$ evolution co-catalyst). EDTA served as sacrificial electron donor in purified water, whereas the photogenerated holes were quenched by water pollutants or chlorides in untreated water media resulting in pollutant utilisation.

\section{Results and Discussions}

\section{Synthesis of CDs}

Pyrolysis of the biomass precursors under air to form the CDs was optimised for high photocatalytic activity, by varying the calcination temperature and time (Fig. S3-S5). First, CDs from purified biomass components were synthesised to correlate their photocatalytic activities with the properties of the pure precursors. As such, $\alpha$ - 
cellulose-, xylan- and lignin-derived CD photoabsorbers were prepared by heating $\alpha$ cellulose, xylan and lignin (Fig. 1a) for four days at 320,250 and $300{ }^{\circ} \mathrm{C}$, respectively (Fig. S3-S4). Next, the potential of $\alpha$-cellulose-rich waste samples for the synthesis of CDs was evaluated by calcinating commercial cotton wool, pads and recyclable cotton fabric (T-shirt) at 320, 340 and $340^{\circ} \mathrm{C}$, respectively (Fig. S1, S5). Cotton-based precursors are excellent for this purpose as they have high cellulose $(\leq 97.7 \%)$ and limited hemicellulose and lignin contents $(\leq 0.5 \%) .{ }^{38}$

To further demonstrate the versatility of our synthetic approach and establish a correlation between biomass composition and CD photocatalytic activity, we employed waste precursors that contain $\alpha$-cellulose, xylan and lignin at various contents. We selected inedible plant waste from Galanthus (G.) nivalis, Garrya ( G.) elliptica, Taxus (T.) baccata, Elaeagnus (E.) X. ebbingei and olive tree (Fig. 1b and Fig. S1) because they are abundant in various climates, have no special growth requirements, and could be obtained at low cost in great quantities. ${ }^{39}$

The optimal conditions for CD synthesis from these waste precursors depend on their relative contents in $\alpha$-cellulose, xylan and lignin as well as their morphologies. ${ }^{40}$ Thermogravimetric analysis (TGA) traces of the plant leaves show three main bands (Table S4 and Fig. S6): xylan degrades first $(210-300 \stackrel{\circ}{ }$ ), followed by $\alpha$-cellulose (303-345 $\stackrel{\circ}{ }$ ), and lignin as a shoulder at higher temperatures (381-434 $\left.{ }^{\circ} \mathrm{C}\right) .41,42$ Olive leaves show additional bands at 460 and $490^{\circ} \mathrm{C}$, possibly due to the presence of other polyphenols in the sample. ${ }^{43}$

Deconvolution of xylan, $\alpha$-cellulose and lignin bands in the TGA plots (Fig. S6), allowed to determine the relative fractional areas (RFAs) for each component (Table S4). The calcination temperature was optimised below the decomposition temperature of the dominating component for each sample, and CDs with the best photocatalytic 
activities were obtained upon the following heat treatments; $230^{\circ} \mathrm{C}$ for $\mathrm{G}$. nivalis, $\mathrm{G}$. elliptica and $T$. baccata, $275^{\circ} \mathrm{C}$ for $E$. X. ebbingei and $290^{\circ} \mathrm{C}$ for olive leaves. Calcination resulted in shrinkage and colour change for all leaves to black, but their overall macroscopic morphologies remained unchanged (Fig. S7).

\section{Characterisation of CDs}

The biomass-derived CDs underwent in-depth spectroscopic, structural and optical characterisation to shed light into their core structures and surface functionalities, which bestow them with distinctive photocatalytic properties. X-ray photoelectron spectroscopy (XPS) suggests that $\alpha$-cellulose CDs consist of graphitic $\mathrm{sp}^{2}$ cores $(\mathrm{C}=\mathrm{C}, 284.8 \mathrm{eV})$, decorated with alkoxy/epoxy $(\mathrm{C}-\mathrm{O}, 286.3 \mathrm{eV})$ and carboxylate functionalities $(\mathrm{C}=\mathrm{O}, 288.5 \mathrm{eV})$, providing them with good solubility in water (Fig. 2a).4, 44, 45 Carboxylates $(531.8 \mathrm{eV}, 89.5 \%)$ are the predominant surface functionalities, which coexist with a smaller number of C-O groups (534.1 eV, 10.5\%; Table S5, entry 1 and Fig. S8). ${ }^{46,47}$ In contrast, the other purified biomass-derived CDs from xylan and lignin, show significantly higher surface coverage by C-O groups (63-70\%) compared to carboxylates (30-37\%) (Table S5 and Fig. S9).

Elemental analysis revealed that CDs from raw biomass contain significant amounts of nitrogen ( $\mathrm{C} / \mathrm{N}$ ratios; $5.5-11)$, due to the $\mathrm{N}$-content of the leaf precursors. The only exception are CDs from cotton wool as the precursor mainly consists of $\alpha$-cellulose (Fig. S10). The N-doping in the waste-derived photoabsorbers, as indicated by XPS, originates from graphitic quaternary (Q) nitrogen $(\mathrm{N}-\mathrm{Q}, \sim 400 \mathrm{eV})$ and pyrrolic functionalities ( 399 eV) for most samples (Fig. 2b, Table S5 and Fig. S11), except for pyridinic ( $398 \mathrm{eV})$ and N-Q groups in G. elliptica- and olive leaf-CDs (Fig. S12). ${ }^{48}$ 
a

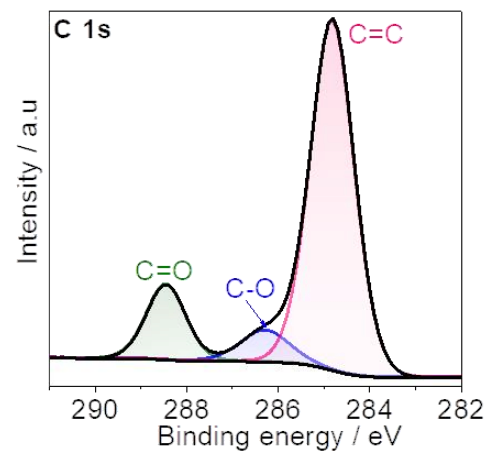

b

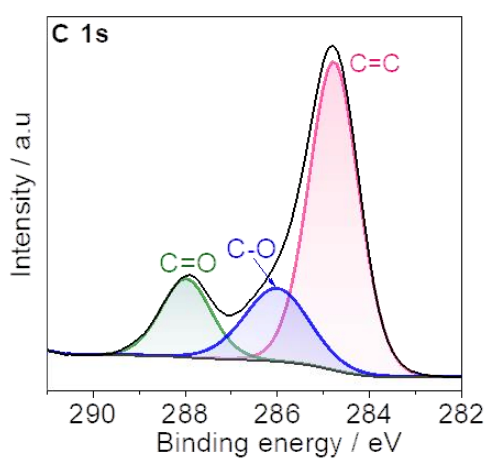

C

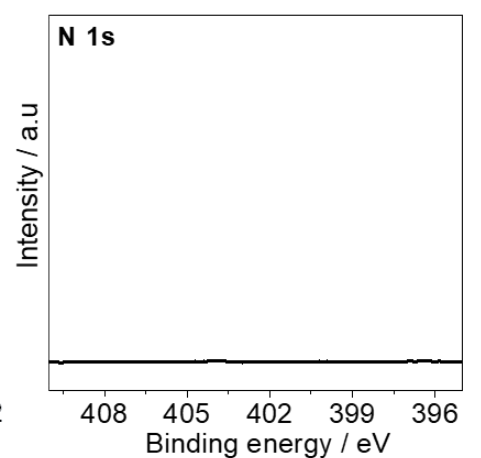

$405 \quad 402 \quad 399$
Binding energy / eV

d

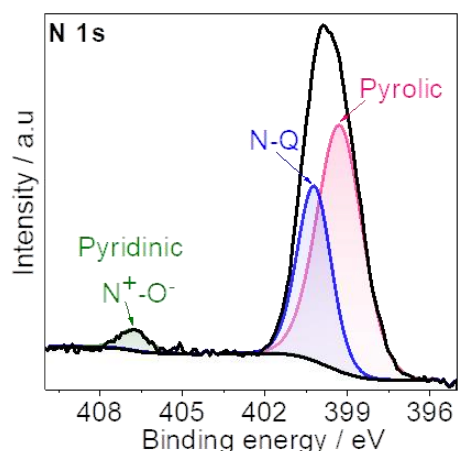

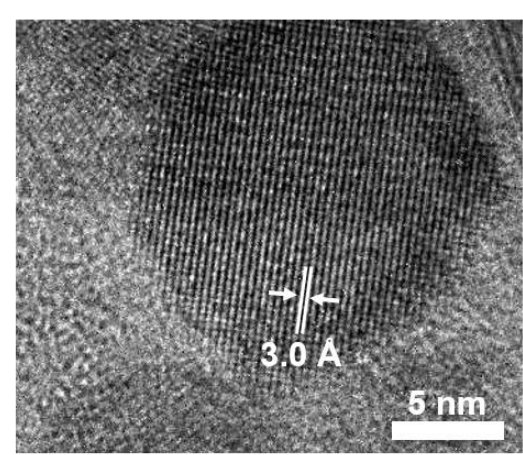

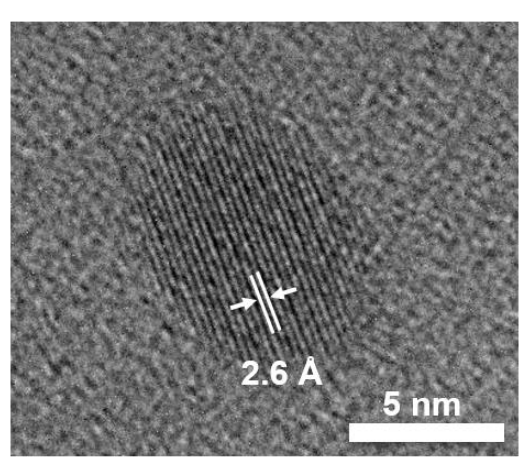

\section{e}

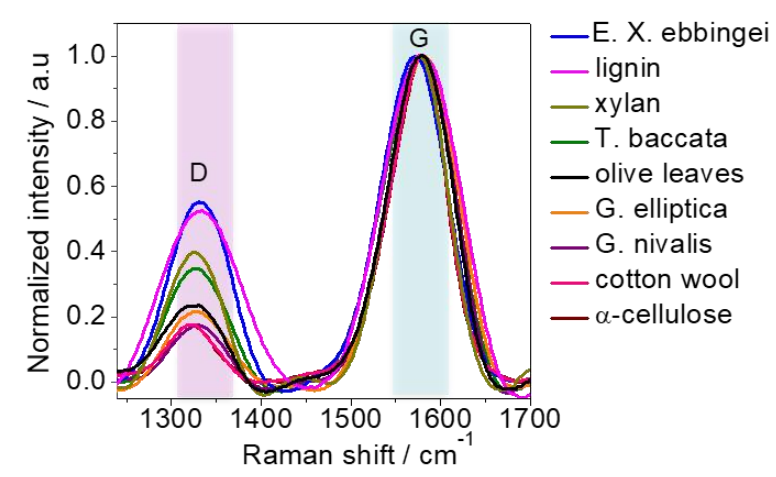

f

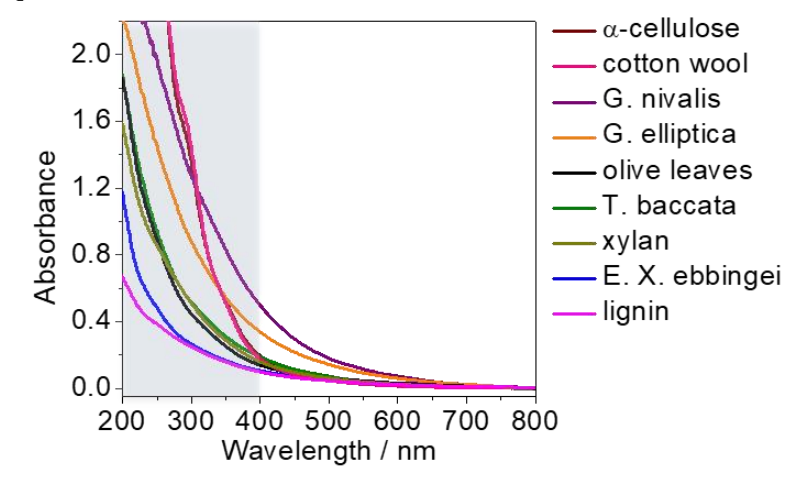

Fig. 2. XPS C 1s and N 1s spectra of $\mathbf{a}, \alpha$-cellulose and b, G. nivalis-derived CDs. The black traces show the 'as recorded' spectra and the coloured plots (pink, blue and green) are the deconvoluted bands for each element. $\mathbf{c}$ and $\mathbf{d}$, TEM images of $\alpha$-cellulose and G. nivalis-derived CDs, respectively. The white lines denote the (100) intralayer spacings. e, Raman spectra of the CDs derived from purified and real-world biomass samples. The intensity was normalised with respect to the G band for all samples. $\mathbf{f}$, UV/vis spectra of CDs dispersed in water $\left(0.1 \mathrm{mg} \mathrm{mL}^{-1}\right)$ at $25^{\circ} \mathrm{C}$. 
Waste-biomass photoabsorbers also show alike $C 1$ s and $O$ 1s environments to CDs from purified biomass (Fig. 2b and Fig. S11, S12), and increased hydrophilicity due to $\mathrm{C}=\mathrm{O}$ groups $(61-79 \%)$. Exception are the photoabsorbers from E. X. ebbingei, which display a higher $\mathrm{C}-\mathrm{O}$ content $(54 \%$, Table S5).

Fourier Transform Infrared (FTIR) spectroscopy also confirms the coexistence of C$\mathrm{OH}, \mathrm{C}=\mathrm{O}$ and $\mathrm{O}-\mathrm{H}$ functionalities at the $\mathrm{CD}$ surfaces $(v=1130 ; 1380$ and $1560,3,4$ $3000-3500 \mathrm{~cm}^{-1}$, respectively), ${ }^{44}$ with skeletal vibrations from the graphitic core tentatively assigned at $1611 \mathrm{~cm}^{-1}$ (Fig. S13). ${ }^{44}$

Transmission electron microscopy (TEM) images show that CDs from $\alpha$-cellulose, cotton wool, G. nivalis and olive leaves are graphitic with average diameters $\leq 11 \mathrm{~nm}$ (Fig. 2c,d, Table S6 and Fig. S14-S16). Lattice fringes allow the assignments of the (100) intralayer spacings; $3.0 \AA$ for $\alpha$-cellulose and $2.6 \AA$ for cotton wool, G. nivalis and olive leaf CDs. The remaining photoabsorbers are of larger sizes $\geq 45 \mathrm{~nm}$ (Table S6 and Fig. S17).

Raman spectroscopy supports low-defect graphitic nanostructures for $\alpha$-cellulose, cotton wool, G. nivalis and olive leaf-derived CDs. Specifically, their D $\left(1331 \mathrm{~cm}^{-1}\right.$, defective $\mathrm{sp}^{2}$ carbon) to $\mathrm{G}\left(1580 \mathrm{~cm}^{-1} \text {, intact } \mathrm{sp}^{2} \text { carbon }\right)^{49}$ band intensity ratios $\left(\mathrm{I}_{\mathrm{D}} / \mathrm{I}_{\mathrm{G}}\right)$, are $\leq 0.21$ (Fig. 2e and Fig. S18), far below those reported for other graphitic CD systems $(\geq 0.75), 4,46,50$ suggesting their higher $\mathrm{sp}^{2}$ character. The remaining photoabsorbers display higher $\mathrm{ID} / \mathrm{IG}_{\mathrm{G}}$ ratios $(\leq 0.55)$, indicating more defective graphitic structures (Fig. 2e).

Powder X-ray diffraction (XRD) shows alike patterns for CDs from $\alpha$-cellulose and cotton wool (Figure S19) perhaps due to the chemical and structural similarities of the organic precursors; broad peaks centred at $27.6^{\circ} 2 \theta$ that correspond to lattice spacings of $3.2 \AA$, consistent with the (002) reflection of graphitic materials $\left(\mathrm{d}_{002}=\right.$ 
3.35). ${ }^{51}$ Photoabsorbers from G. Nivalis, G.elliptica, T. baccata and E. X. ebbingei exhibit broad bands with maxima at $25.3^{\circ} 2 \theta$, which indicate interlayer spacings of 3.5 $\AA$. The low signal-to-noise ratio for $T$. baccata- and $E$. X. ebbingei-derived materials is indicative of their more amorphous nature compared to the rest samples.

${ }^{13} \mathrm{C}$ nuclear magnetic resonance (NMR) studies further support predominant $\mathrm{sp}^{2}$ core environments for the CDs from $\alpha$-cellulose, cotton wool, G. nivalis and olive leaves with peaks between 110 and 150 ppm $^{4,52}$ (Fig. S20). Surface carboxylate groups were also detected (160-180 ppm), whereas the absence of signals between 10 and 70 ppm, supports limited $\mathrm{sp}^{3}$ character in these CDs. The other photoabsorbers exhibit higher $\mathrm{sp}^{3}$ character than $\alpha$-cellulose-, cotton wool-, olive leaf- and G. nivalis-derived CDs (Fig. S20).

The UV-vis absorption spectra (Fig. 2f) of all photoabsorbers show strong absorption in the UV region with featureless tails extending into the visible region. ${ }^{4}$ These properties are assigned to $\pi-\pi^{*}(\mathrm{C}=\mathrm{C}$, core $)$ and $\mathrm{n}-\pi^{\star}(\mathrm{C}=\mathrm{O}$, surface $)$ transitions of the carbonaceous structures. ${ }^{3}$ Higher graphitic character results in enhanced absorption, ${ }^{4}$ as indicated by the stronger absorption profiles of $\alpha$-cellulose-, cotton wool- and $G$. nivalis-derived CDs, compared to the other photoabsorbers.

\section{Photocatalysis with CDs in purified water}

CDs from purified biomass components and biomass waste were used as photoabsorbers to generate $\mathrm{H}_{2}$ in the presence of EDTA $(0.1 \mathrm{M}, \mathrm{pH} 6,3 \mathrm{~mL})$ as SED and $\mathbf{N i P}(50 \mathrm{nmol})$ as the $\mathrm{H}_{2}$ evolution co-catalyst. All photocatalytic experiments were carried out upon irradiation with simulated solar light (AM $1.5 \mathrm{G}, 100 \mathrm{~mW} \mathrm{~cm}^{-2}$ ), under $\mathrm{N}_{2}$ atmosphere $\left(2 \% \mathrm{CH}_{4}\right.$ as internal gas chromatography standard) at $25^{\circ} \mathrm{C}$. Gas samples of the headspace $(20 \mu \mathrm{L})$ were taken from the photoreactor (total volume, 7.7 
$\mathrm{mL}$ ), and analysed periodically by gas chromatography to quantify $\mathrm{H}_{2}$. The results after 6 and $24 \mathrm{~h}$ of irradiation are discussed below.

The quantity of CDs was first systematically varied between 0.03 and $2.8 \mathrm{mg}$ (Fig. 3a and Fig. S21) to optimise the system's performance for maximum $\mathrm{H}_{2}$ yield (in $\mu \mathrm{mol}$ ), from which the specific activities $\left(\mu \mathrm{mol} \mathrm{H} \mathrm{H}_{2}\left(\mathrm{gCDs}_{\mathrm{C}}\right)^{-1} \mathrm{~h}^{-1}\right)$ were determined. NiP turnover numbers (TONNiP, mol H2 $\left(\mathrm{mol} \mathrm{NiP}^{-1}\right.$ ) were calculated at $50 \mathrm{nmol} \mathbf{N i P}$ (Table S7). $\alpha$-Cellulose CDs $(2.2 \mathrm{mg})$ showed the highest $\mathrm{H}_{2}$ yield $(15.6 \pm 0.7 \mu \mathrm{mol})$ and TONNiP (312.8 \pm 14.6$)$ among all CDs synthesised from purified biomass components after 24 h of irradiation (Fig. 3a and Fig. S21). A benchmark specific activity of $13,450 \mu \mathrm{mol}$ $\mathrm{H}_{2}(\mathrm{gcDs})^{-1} \mathrm{~h}^{-1}$ was achieved with $0.03 \mathrm{mg} \alpha$-cellulose CDs (Fig. 3b) after $1 \mathrm{~h}$ of irradiation, which outperforms previously reported carbonaceous photocatalysts (CDs and carbon nitrides), when combined with noble-metal free co-catalysts (activities $\leq$ $7,950 \mu \mathrm{mol} \mathrm{H} 2\left(\mathrm{gCDs}^{-1} \mathrm{~h}^{-1}\right.$, Table S8). ${ }^{2-4,53}$ It is also higher than what has been reported for numerous benchmark non-carbonaceous and/or multi-absorber systems in purified water (19-12,000 $\mu \mathrm{mol} \mathrm{H} \mathrm{H}^{-1} \mathrm{~h}^{-1}$, Table S2), which serve $\mathrm{H}_{2}$ evolution and pollutant degradation separately, under $\mathrm{N}_{2}$ and $\mathrm{O}_{2}$, respectively. ${ }^{54-61} \mathrm{The}_{2}$ production rate drops after $6 \mathrm{~h}$ of irradiation for all photoabsorbers, due to instability and decomposition of the molecular framework of the NiP catalyst (Fig. 3a). ${ }^{62}$

Control experiments in the absence of CDs or NiP resulted in no $\mathrm{H}_{2}$ generation, indicating that $\alpha$-cellulose CDs themselves are not catalytic for proton reduction. Without EDTA, negligible $\mathrm{H}_{2}$ was generated, suggesting insignificant $\mathrm{CD}$ autooxidation and the necessity for a SED (Fig. S22a and Table S9).

During photocatalysis under visible-light irradiation only $(\lambda>400 \mathrm{~nm})$, in the presence of EDTA and NiP, $\alpha$-cellulose CDs showed lower (28\%) but still persistent 
photocatalytic fuel production, suggesting their good activity as visible-light photoabsorbers (Table S9 and Fig. S22b).

a

b
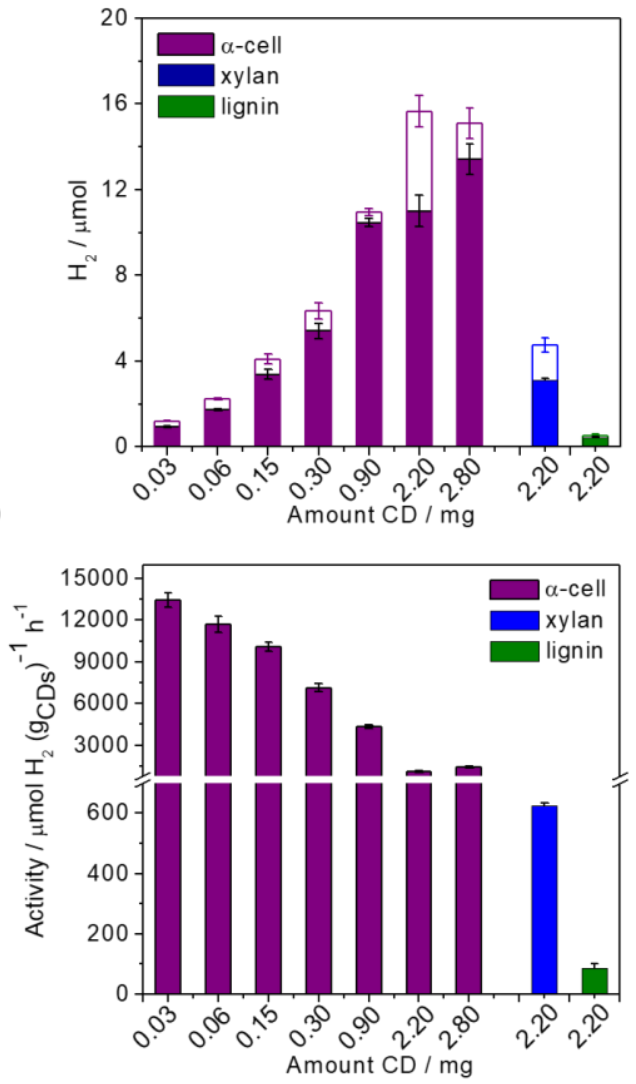

C
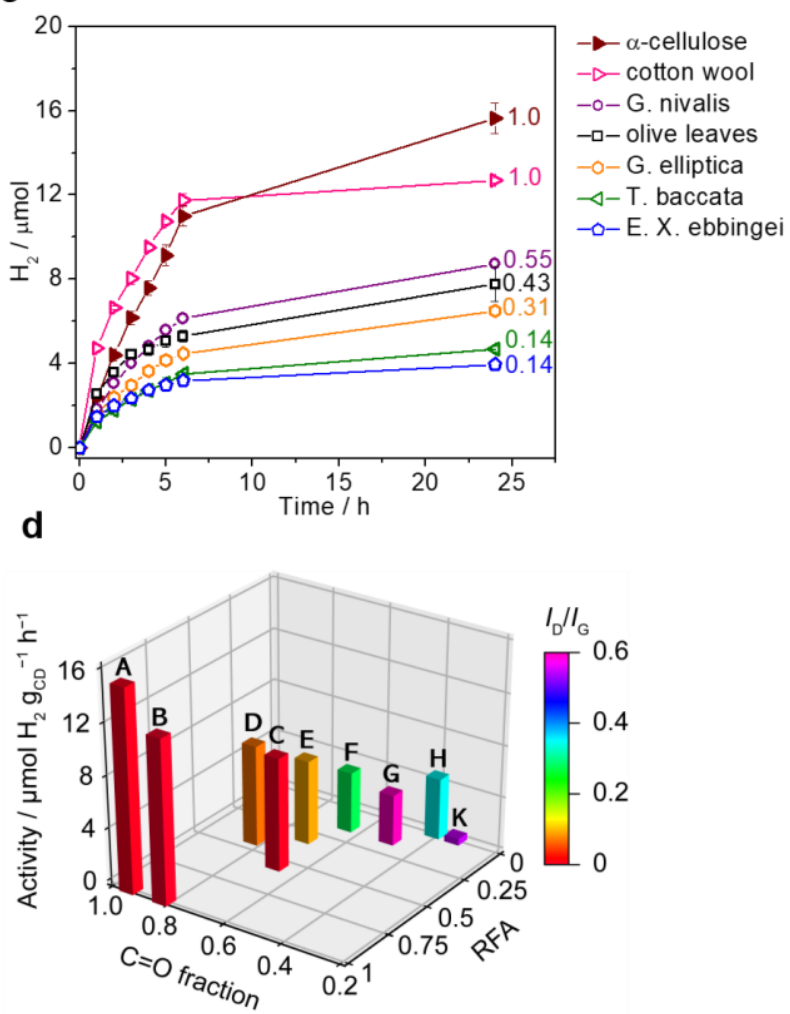

Fig. 3. a, $\mathrm{H}_{2}$ produced (in $\mu \mathrm{mol}$ ) from $\alpha$-cellulose, xylan and lignin-derived photoabsorbers after $6 \mathrm{~h}$ (solid bars) and $24 \mathrm{~h}$ (empty bars) of irradiation, in purified water in the presence of EDTA and NiP. Error bars indicate the standard deviation as derived from three measured samples from each CD system. $\mathbf{b}$, Maximum specific activities of $\alpha$-cellulose, xylan and lignin derived light-harvesters after $1 \mathrm{~h}$ of irradiation. c, $\mathrm{H}_{2}$ production (in $\mu \mathrm{mol}$ ) from waste biomass photoabsorbers after $24 \mathrm{~h}$ of irradiation in purified water in the presence of EDTA and NiP. For comparison, the hydrogen yield of $\alpha$-cellulose CDs is shown (red close symbols) under the same conditions. The numbers on the right, indicate the RFAs of $\alpha$-cellulose in all leaf precursors used for the CD synthesis. $\mathbf{d}$, 4D plot which correlates the photocatalytic properties of CDs with the RFAs of their precursor components and their structural properties, such as their graphitic characters $\left(\mathrm{I}_{\mathrm{D}} / \mathrm{I}_{\mathrm{G}}\right)$ and fractions of surface carboxylate $(\mathrm{C}=0)$ groups; $\mathrm{A}: \alpha$ cellulose, B: cotton wool, C: G. Nivalis, D: Olive leaves, E: G. Elliptica, F: T. bacatta, G: E. X. ebbingei, $\mathrm{H}$ : xylan and $\mathrm{K}$ : lignin. Conditions for photocatalytic experiments in a, b and c; CDs were irradiated with simulated solar light (AM 1.5G, $100 \mathrm{~mW} \mathrm{~cm}^{-2}, 25$ $\left.{ }^{\circ} \mathrm{C}\right)$ for 6 and $24 \mathrm{~h}$, in EDTA $(0.1 \mathrm{M}, \mathrm{pH} 6,3 \mathrm{~mL})$ with NiP (50 nmol), under $\mathrm{N}_{2}$ atmosphere with $2 \% \mathrm{CH}_{4}$.

Xylan- and lignin-derived photoabsorbers in EDTA solutions yielded $4.7 \pm 0.3$ and 0.5 $\pm 0.1 \mu \mathrm{mol} \mathrm{H}$ after $24 \mathrm{~h}$ irradiation (Fig. 3a and Table S7), and maximum specific 
activities of 625 and $87 \mu \mathrm{mol} \mathrm{H} 2\left(\mathrm{gCDs}^{-1} \mathrm{~h}^{-1}\right.$ after $1 \mathrm{~h}$ of irradiation, respectively (Fig. 3b). The distinctive photocatalytic properties of CDs from different purified biomass components are presumably associated with their unique chemical compositions, architectures and microstructures (Fig. 1a). $\alpha$-cellulose is a linear homopolymer that packs into insoluble fibrils which are partially crystalline, whereas xylan is an amorphous branched polysaccharide. Lignin is also amorphous consisting of randomly cross-linked phenolic units. ${ }^{9}$ As such, the pyrolysis of more crystalline polymers of regular microstructures, such as $\alpha$-cellulose, might support the formation of CDs with improved photocatalytic performances.

Using biomass waste for CD synthesis enables an economically viable approach to synthesise photocatalysts for sustainable fuel production. As a demonstration, we tested the photocatalytic performances of CDs from commercial cotton and readily available plant leaves. Although cotton wool-derived CDs showed almost identical photocatalytic activity to $\alpha$-cellulose CDs $\left(11.8 \pm 0.3 \mu \mathrm{mol} \mathrm{H} \mathrm{H}_{2}\right.$ after $6 \mathrm{~h}$; Fig. 3c and Table S10), given the similar nature of their precursors, photoabsorbers from cotton pads and a T-shirt are less active ( $4.9 \pm 0.2$ and $1.7 \pm 0.2 \mu \mathrm{mol} \mathrm{H}_{2}$, respectively, $6 \mathrm{~h}$ ). This is presumably due to additives in the commercial materials (Table S10 and Fig. S5, S23).

Photoabsorbers from plants, due to the composite nature of the precursors (Fig. 1b), showed lower $\mathrm{H}_{2}$ evolution yields than $\alpha$-cellulose CDs. The best performances were observed for CDs from $G$. nivalis and olive leaves $\left(6.2 \pm 0.1\right.$ and $5.3 \pm 0.3 \mu \mathrm{mol} \mathrm{H}_{2}$, respectively, 6h), which are consistent with their highest RFAs for $\alpha$-cellulose, and lowest for xylan and lignin (Fig. 3c and Tables S4, S10). The $\mathrm{H}_{2}$ evolution yields for the remaining photoabsorbers declined as the RFA of $\alpha$-cellulose in their precursors was reduced. As such, light-harvesters from T. baccata and E. X. ebbingei with 
minimum $\alpha$-cellulose RFAs, show the lowest $\mathrm{H}_{2}$ yields $(3.5 \pm 0.1$ and $3.2 \pm 0.2 \mu \mathrm{mol}$ $\mathrm{H}_{2}$, respectively, 6 h; Fig. $\mathbf{3 c}$ and Tables S4, S10).

The correlation between the RFAs of the precursor components and the photocatalytic performances of the derived photoabsorbers, gives therefore for the first time a qualitative predictor for photocatalytic efficiencies of lignocellulosic-derived CDs (Table S11 and Figure 3d). Also, a correlation between the photocatalytic activities of the synthesised CDs and their structural properties is observed; the best properties are associated with smaller size and more graphitic CD cores (lower $\left.\mathrm{I}_{\mathrm{D}} / \mathrm{I}_{\mathrm{G}}\right)$, and higher surface coverage by carboxylate groups (Figure 3d).

\section{Coupled photocatalysis and pollutant utilisation with CDs}

A common limitation in photocatalysis is the requirement for (I) pure water to avoid catalyst poisoning at high salinities and/or impurity content, ${ }^{27}$ and (ii) an external and expensive SED. ${ }^{23}$ Given the global shortage of clean water supplies, ${ }^{24,} 25$ and challenges of water purification in many developing countries, we sought to establish the photocatalytic activity of our biomass-derived CDs in untreated sea (Gulf of Mexico) and river water (river Cam), first in the presence and subsequently in the absence of EDTA (Fig. 4).

Simulated solar irradiation of $\alpha$-cellulose CDs in sea and river water, with NiP and EDTA $(0.1 \mathrm{M}, \mathrm{pH} 6,3 \mathrm{~mL})$, resulted in a slight improvement in the photocatalytic activity ( 15\%) compared to purified water $(12.6 \pm 0.6 \mu \mathrm{mol} \mathrm{H}, 6$ h, Fig. 4a and Tables S12, S13).

Control experiments using $\alpha$-cellulose CDs without NiP but in the presence of EDTA, resulted in no $\mathrm{H}_{2}$ evolution in untreated water, suggesting that no species (i.e. metal traces) exist in raw water that could drive proton reduction (Table S9 and Fig. S24). 
a

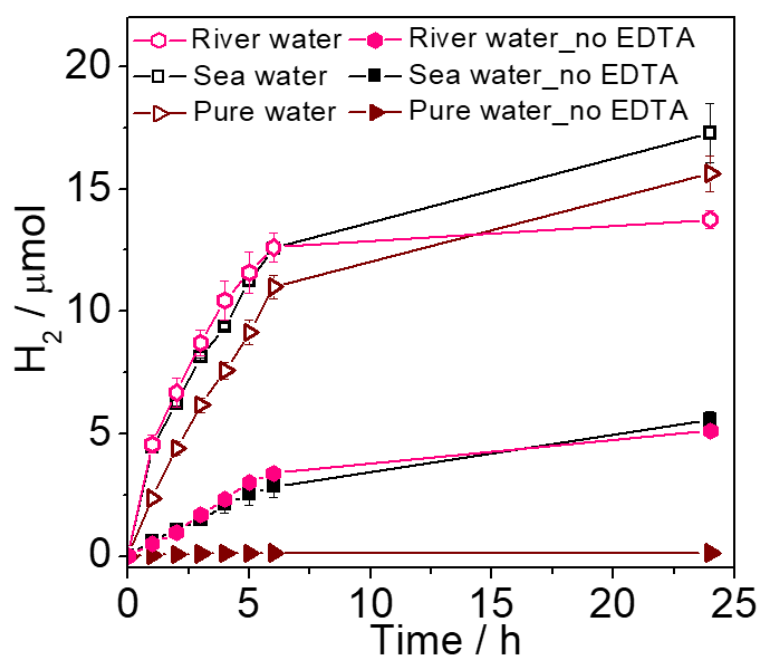

C

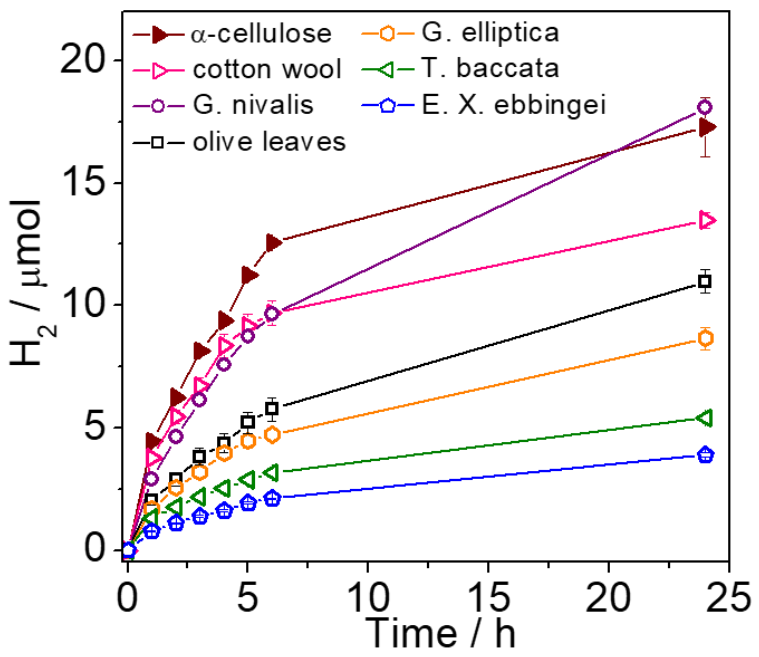

b

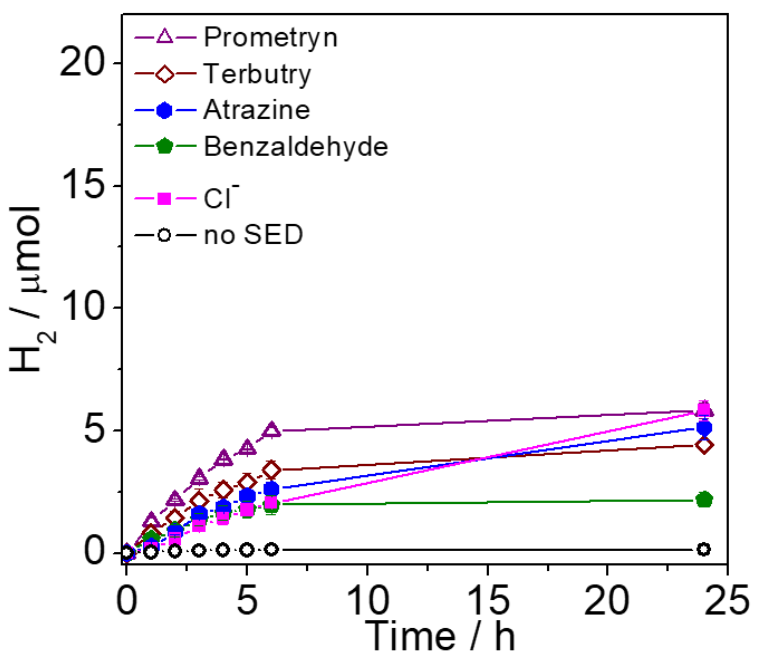

d

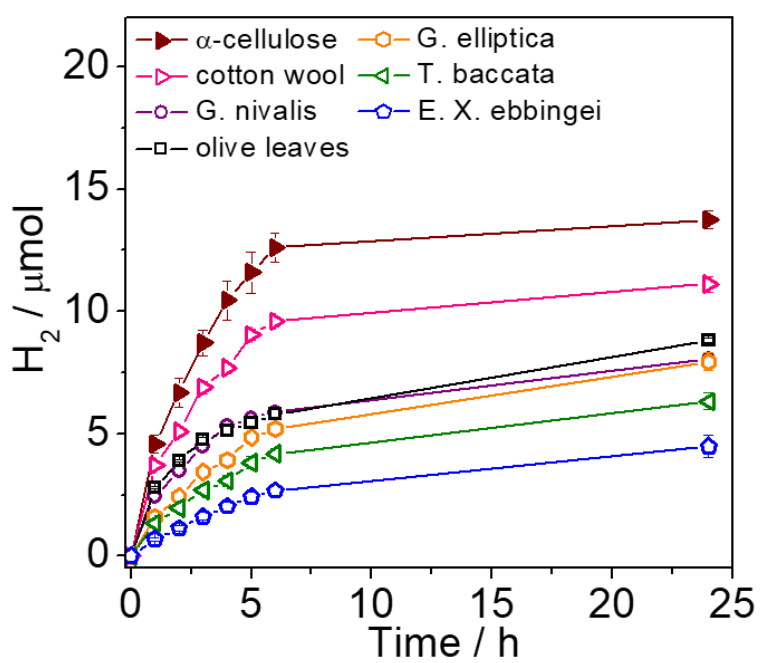

Fig. 4. a, Photo- $\mathrm{H}_{2}$ production with $\alpha$-cellulose CDs in the absence (filled symbols) and presence (open symbols) of EDTA $(0.1 \mathrm{M}, \mathrm{pH} 6,3 \mathrm{~mL}) . \mathbf{b}$, Solar- $\mathrm{H}_{2}$ generation using $\alpha$-cellulose CDs in $\mathrm{KP}_{\mathrm{i}}(0.1 \mathrm{M}, \mathrm{pH} 6,3 \mathrm{~mL})$, in the absence of EDTA, but upon addition of organic pollutants $(200 \mu \mathrm{mol})$ and chloride ions (3.2 wt\%) found in river (open symbols) and sea water (filled symbols). The results in the absence of any electron donor are also provided for comparison. c and $\mathbf{d}$, Photo- $\mathrm{H}_{2}$ production in sea and river water, respectively, using CDs form $\alpha$-cellulose and biomass waste, in the presence of EDTA (0.1 M, pH 6, $3 \mathrm{~mL})$. All experiments were carried out under full solar light irradiation (AM $1.5 \mathrm{G}, 100 \mathrm{~mW} \mathrm{~cm}^{-2}$ ), for $24 \mathrm{~h}$, in the presence of CDs (2.2 mg) and $\mathrm{NiP}(50 \mathrm{nmol})$, under $\mathrm{N}_{2}$ atmosphere containing $2 \% \mathrm{CH}_{4}$, at $25{ }^{\circ} \mathrm{C}$. Error bars indicate the standard deviations as derived from three measured samples from each CD system.

However, without EDTA but in the presence of NiP, $\alpha$-cellulose CDs after $6 \mathrm{~h}$ photogenerated $2.9 \pm 0.5$ and $3.4 \pm 0.2 \mu \mathrm{mol} \mathrm{H}_{2}$ in sea and river water $(\mathrm{pH} 6)$, 
respectively. These activities deviate significantly from those of the $\alpha$-cellulose CD/NiP system in purified water, under the same conditions, where negligible $\mathrm{H}_{2}$ production was observed (Fig. 4a and Table S14). This indicates that pollutants present in the untreated aqueous media, act as SEDs to extract photogenerated holes. ${ }^{4}$ Untreated sea water contains a cocktail of organic compounds, ${ }^{63}$ among which benzaldehyde and atrazine could act as SEDs (Fig. S25). Chloride anions that are abundant in seawater could also serve the same role. ${ }^{64}$ Similarly, untreated river water is rich in organic herbicides/pesticides, ${ }^{65}$ among which prometryn and terbutryn can quench the photogenerated holes (Fig. S25).

To confirm this, an aqueous $\alpha$-cellulose CD phosphate solution $(\mathrm{KP}, 0.1 \mathrm{M}, \mathrm{pH} 6,3$ $\mathrm{mL}$ ), without EDTA, was bestowed with these species (200 $\mu \mathrm{mol})$, in the presence of NiP. After $6 \mathrm{~h}$ of irradiation (AM 1.5 G, $100 \mathrm{~mW} \mathrm{~cm}^{-2}$ ), $2.1 \pm 0.2$ to $5.0 \pm 0.2 \mu \mathrm{mol} \mathrm{H} 2$ was produced (Fig. 4b, Table S15 and Fig. S26a), which is close to that obtained in untreated water, in the absence of EDTA (Fig. 4a). As such, the unwanted toxic substances and/or $\mathrm{Cl}^{-}$, do not only allow unhindered photocatalytic performances, but also eliminate the need for additional expensive SEDs, facilitating simultaneously fuel synthesis and pollutant utilisation. This is perhaps due to good affinities between the $\mathrm{CDs}(\mathrm{OH}$ groups) and the pollutants which favour closer proximities and possibly improved hole transfer and thus substrate oxidation. ${ }^{25}$

To the best of our knowledge, this is the first time that photogenerated CD holes, drive the oxidation of pollutants in untreated water (neutral $\mathrm{pH}$ ), simultaneously with $\mathrm{H}_{2}$ generation, using a noble-metal free co-catalyst. Such an overall process has been rarely reported in raw water, but instead in extremely acidic synthetic water, enriched with pollutants. $\mathrm{TiO}_{2}$-based UV-light harvesters or multi-absorber systems, in combination with noble catalysts $(\mathrm{Au}, \mathrm{Pt})$ have been employed (Table S1)..$^{28-30}$ 
However, such systems ignore attenuated photoabsorption effects (originated from background organics in untreated water) which could also inhibit photocatalysis, and also competitive organics over pollutant adsorption onto the photocatalytic components that suppresses pollutant utilisation. ${ }^{31}$

Finally, the photocatalytic performances of CDs from biomass waste, were also evaluated in untreated water in the presence of EDTA and NiP. In sea water, after 24 $\mathrm{h}$ of irradiation, all CDs showed higher photocatalytic performances than in purified water, under the same conditions (Fig. 4c, Table S12 and Fig. S26b). On the other hand, all systems show slightly lower performances in river compared to sea water (Fig. 4d, Table S13 and Fig. S26b). These results suggest the robust characters of our photocatalytic systems, which maintain their good photocatalytic properties, independently of the purity and origin of the aqueous phase. As such, the toxic water contaminants do not restrict the performances of the CDs by hindering photoabsorption and/or electron transfer, and more remarkably do not poison the molecular NiP catalyst. Instead, the photocatalytic performances of our systems (17.3 $\mu \mathrm{mol} \mathrm{H} \mathrm{H}_{2}, 13,660 \mu \mathrm{mol} \mathrm{H}_{2}\left(\mathrm{gcDs}^{-1} \mathrm{~h}^{-1}\right.$, Table S12, entries 2,3), even in untreated water, remain the highest among metal-free photoabsorbers combined with precious metalfree catalysts (Table S8). ${ }^{2-4,53}$ They are also tremendously higher than those of the state-of-art $\mathrm{TiO}_{2}-\mathrm{Pt}$ systems $\left(\leq 500 \mu \mathrm{mol} \mathrm{H} \mathrm{H}_{2}\left(\mathrm{gCDs}^{-1} \mathrm{~h}^{-1}\right)\right.$ which couple pollutant degradation to fuel production under benign conditions $(\mathrm{pH}$ 6, Table S1, entries 13). ${ }^{28-30,66}$ They also outperform other dual-function photocatalytic systems $(2-1,500$ $\mu$ mol H $\mathrm{g}^{-1} \mathrm{~h}^{-1}$, Table S1, entries 4-9) which use either $\mathrm{TiO}_{2}$ alone or combined with visible light-harvesters, such as CdS and/or carbon nitride to boost photoabsorption. ${ }^{32}$, $33,35,67-69$ The only exception is a $\mathrm{MoS}_{2}-\mathrm{TiO}_{2}$ systems for which overestimated activities up to $380,000 \mu \mathrm{mol} \mathrm{H} \mathrm{g}^{-1} \mathrm{~h}^{-1}$ were reported (Table S1, entry 10), upon 
neglecting the mass of the $\mathrm{TiO}_{2}$ component. ${ }^{36}$ It is also worth noting that $\alpha$-cellulose CDs show activities about 500 times higher compared to a $\mathrm{TiO}_{2}-\mathrm{Au}$ system which is also operated in untreated water (28 $\mu \mathrm{mol} \mathrm{H} \mathrm{g}^{-1} \mathrm{~h}^{-1}$, Table S1, entry 8) instead of synthetic water. ${ }^{69}$

\section{Conclusions}

A photocatalytic system based on CDs derived from raw biomass waste and a $\mathrm{Ni}$ cocatalyst has been established for simultaneous $\mathrm{H}_{2}$ production and pollutant utilisation. This approach creates new perspectives as it allows the production of low-cost and non-toxic CD photocatalysts from lignocellulosic waste to convert polluted river and sea water into energy carriers on virtually any inhabited location worldwide. Water pollution does not affect the versatile character of the presented systems, but instead works to their advantage by eliminating the need for external SEDs through pollutant oxidation. This waste-derived energy production-system operates at room temperature and neutral $\mathrm{pH}$, using a single-atom photocatalyst without the need for noble-metal co-catalysts and synthetic wastewater to achieve high performances. This solar process encourages the development of scalable and economically sustainable dual-function photocatalytic systems.

\section{Experimental Section}

Materials. The purified biomass components, $\alpha$-cellulose, xylan and lignin, as well as natural sea water (from Gulf of Mexico) and EDTA were purchased from Sigma-Aldrich and used without any further purification. Cotton wool, cotton pads and a cotton T-shirt were purchased from local merchants in Cambridge, UK. The crude plant biomass samples, G. nivalis, G. elliptica, T. baccata and E. X. ebbingei were collected from the 
gardens of St John's College, University of Cambridge, UK, whereas the olive leaves were sourced from Trachoni, Cyprus. The untreated river water was obtained from the river Cam (Midsummer Park), Cambridge, UK.

CD synthesis. All organic precursors were calcinated in muffle Carbolite furnaces for 4 days in air, at optimised temperatures. $\alpha$-Cellulose and cotton wool were treated thermally at $320^{\circ} \mathrm{C}$, xylan at $250{ }^{\circ} \mathrm{C}$, lignin at $300{ }^{\circ} \mathrm{C}$, cotton pads and T-shirt at 340 ${ }^{\circ} \mathrm{C}$, G. nivalis, G. elliptica and T. baccata at $230^{\circ} \mathrm{C}$, E. X ebbingei at $275^{\circ} \mathrm{C}$ and olive leaves at $290^{\circ} \mathrm{C}$. All precursors $(10 \mathrm{~g})$ were calcinated in glass vessels, without any pretreatment. The samples after carbonization were grounded into fine powders and used in their crude form for all types of characterisation and photocatalytic experiments.

Nickel bis(diphosphine) (NiP) synthesis. The hydrogen evolution co-catalyst, NiP, was synthesised and characterised as reported previously. ${ }^{70}$

Structural, spectroscopic and optical characterisation. XPS characterisation of CDs was performed on a K-Alpha spectrometer (Thermo Scientific, East Grinstead, UK), utilising a monochromatic Al Ka X-ray source (1486.6 eV, $400 \mu \mathrm{m}$ spot size, 36 W). Samples were prepared by drop casting the CD aqueous solutions $\left(2 \mathrm{mg} \mathrm{mL}^{-1}\right)$ on clean fluorine-doped indium tin oxide (FTO)-coated glass slides $(2 \times 1 \mathrm{~cm})$, and drying at room temperature, under atmospheric pressure. The spectra were collected at NEXUS (Newcastle University, UK). High resolution spectra were recorded at a pass energy of $40 \mathrm{eV}$. Each sample was measured at 3 different points with similar results. The data were processed using CasaXPS.

FTIR spectra were recorded on a ThermoScientific Nicolet iS50 FTIR spectrometer equipped with an Attenuated Total Reflectance (ATR) accessory, in the range of 400 to $4000 \mathrm{~cm}^{-1}$, and analysed using the Omnic software. Raman spectra were acquired 
on a Horiba LabRAM HR Evolution system using a $473 \mathrm{~nm}$ laser. ${ }^{13} \mathrm{C}$ NMR spectra for all CD samples were measured using a $400 \mathrm{MHz}$ Bruker NMR spectrometer in $\mathrm{D}_{2} \mathrm{O}$. All samples were prepared upon dispersing $150 \mathrm{mg}$ of the $\mathrm{CDs}$ in $0.7 \mathrm{~mL} \mathrm{D}_{2} \mathrm{O}$. The optical properties of the CDs $\left(0.1 \mathrm{mg} \mathrm{mL}^{-1}\right)$ were investigated with a Varian Cary 50 UV/vis spectrophotometer, in the wavelength range between 250 and $800 \mathrm{~nm}$ (in water), using quartz cuvettes with $1 \mathrm{~cm}$ pathlength. The TEM images of the carbonaceous materials were obtained using a JEOL JEM-2011 electron microscope equipped with a Gatan 794 CCD camera, and fitted with a LaB6 filament, operating at an accelerating voltage of $250 \mathrm{kV}$. For this purpose, a droplet from the aqueous solutions of the samples was placed on a 300 mesh copper grid containing a holey carbon film and dried under atmospheric pressure. TGA measurements were performed with a Mettler Toledo TGA analyser, from 50 to $800^{\circ} \mathrm{C}$ at a heating rate of $10^{\circ} \mathrm{C} \min ^{-1}$ under $\mathrm{N}_{2}$ flow.

Photocatalytic experiments. The samples for photocatalytic experiments were prepared in borosilicate glass vials $(7.7 \mathrm{~mL})$ by first dispersing the as calcinated $\alpha$ cellulose CDs $(0.03-2.80 \mathrm{mg})$ and $\mathbf{N i P}(50 \mathrm{nmol}, 200 \mu \mathrm{L}, 0.25 \mathrm{mM})$ in aqueous EDTA solutions $(0.1 \mathrm{M}, 3 \mathrm{~mL}, \mathrm{pH} 6)$. The vials were subsequently sealed with rubber septa (Subaseal), purged with $\mathrm{N}_{2}$ containing $2 \% \mathrm{CH}_{4}$ (internal gas chromatography standard) for about $20 \mathrm{~min}$ and irradiated using a Newport Oriel solar light simulator (100 mW cm${ }^{-2}$ ) equipped with an air mass $1.5 \mathrm{global}$ filter (AM 1.5G), unless otherwise stated. Photocatalytic experiments under visible-light irradiation only, were carried out in the presence of a longpass filter $(\lambda>400 \mathrm{~nm})$. The samples were kept at $25^{\circ} \mathrm{C}$ and stirred constantly during irradiation. Samples of the headspace gas $(20 \mu \mathrm{L})$ were taken from the photoreactor and analysed by gas chromatography at an hourly basis for the first $6 \mathrm{~h}$ and after $24 \mathrm{~h}$. For this purpose, an Agilent 7890A gas chromatograph, 
equipped with a $5 \AA$ molecular sieve column and a thermal conductivity detector (TCD) was used.

The photocatalytic performances of the remaining CDs were evaluated as described above, upon dispersing $2.2 \mathrm{mg}$ of the as calcinated CDs along with $\mathrm{NiP}(50 \mathrm{nmol})$ in EDTA solutions $(0.1 \mathrm{M}, 3 \mathrm{~mL}, \mathrm{pH}$ 6). For the investigation of the photocatalytic activities of the CDs in sea and river water, the aqueous phases were first passed through a $0.20 \mu \mathrm{m}$ filter, to remove any solids and/or microorganisms, prior the addition of EDTA.

Photocatalytic experiments in purified water in the presence of organic pollutants (200 $\mu \mathrm{mol}$ ) and chloride ions (3.2 wt\%, same concentration as sea water), were carried out by dispersing the $\alpha$-cellulose CDs (2.2. $\mathrm{mg})$ in phosphate solution $\left(\mathrm{KP}_{\mathrm{i}}, 0.1 \mathrm{M}, \mathrm{pH} 6,3\right.$ $\mathrm{mL})$, without EDTA, in the presence of $\mathbf{N i P}(50 \mathrm{nmol})$.

Data treatment. All photocatalytic experiments were carried out as triplicates, and the derived performances are reported as mean values \pm standard deviations $(\sigma)$.

Internal Quantum Efficiency (IQE) determination. $\mathrm{H}_{2}$ evolution was driven upon irradiation of an $\mathrm{O}_{2}$-free solution of the $\alpha$-cellulose CDs with monochromatic light $(\lambda=$ $360 \mathrm{~nm}$ and light intensity (I) of $4.05 \mathrm{~mW} \mathrm{~cm}^{-2}$ ) produced by a solar simulator (LOT LSN 254) equipped with a monochromator (LOT MSH 300). IQE was determined using equation 1 (eq. 1), where $n_{\mathrm{H}_{2}}$ is the moles of photoproduced $\mathrm{H}_{2}$, tirr the irradiation time (in s), $A$ the irradiation cross-section (in $\mathrm{cm}^{2}$ ), $\alpha$ the percentage of absorbed light, $N_{A}$ and $h$ are the Avogadro and Planck constants, respectively, and $c$ the speed of light.

$$
\operatorname{IQE}(\%)=\frac{\left(2 \cdot n_{\mathrm{H}_{2}} \cdot N_{A} \cdot h \cdot \mathrm{c}\right)}{t_{i r r} \cdot \lambda \cdot I \cdot A \cdot \alpha} \cdot 100
$$




\section{Conflicts of interest}

A patent application on the reported work has been filed by Cambridge Enterprise (UK patent application number GB1808905.2) which lists DSA, HK and ER as the inventors.

\section{Acknowledgements}

This work was financially supported by the Christian Doppler Research Association (Austrian Federal Ministry for Digital and Economic Affairs and the National Foundation for Research, Technology and Development) and OMV. Dr. Heather F. Greer is greatly thanked for obtaining the TEM images. Dr. Xin Fang is acknowledged for valuable help with artwork preparation, and for helpful discussions along with Dr. Katarzyna P. Sokol, that improved the manuscript. Mr. Arjun Vijeta is thanked for help in collecting the plant species from the gardens of St John's College, Cambridge. Sotiris and Elena Achilleos are acknowledged for providing olive leaves. The Environmental agency is thanked for providing information regarding the contents of the water sample collected from river Cam.

\section{References}

1. P. V. Kamat and J. Bisquert, J. Phys. Chem. C, 2013, 117, 14873-14875.

2. G. A. M. Hutton, B. C. M. Martindale and E. Reisner, Chem. Soc. Rev., 2017, 46, 6111-6123.

3. B. C. M. Martindale, G. A. M. Hutton, C. A. Caputo and E. Reisner, J. Am. Chem. Soc., 2015, 137, 6018-6025.

4. B. C. M. Martindale, G. A. M. Hutton, C. A. Caputo, S. Prantl, R. Godin, J. R. Durrant and E. Reisner, Angew. Chem. Int. Ed., 2017, 56, 6459-6463. 
5. H. Yu, R. Shi, Y. Zhao, G. I. N. Waterhouse, L.-Z. Wu, C.-H. Tung and T. Zhang, Adv. Mater., 2016, 28, 9454-9477.

6. J. Kim, S. H. Lee, F. Tieves, D. S. Choi, F. Hollmann, C. Paul and C. B. Park, Angew. Chem. Int. Ed., 2018, 130, 14021-14024.

7. L. Cao, S. Sahu, P. Anilkumar, C. E. Bunker, J. Xu, K. A. S. Fernando, P. Wang, E. A. Guliants, K. N. Tackett and Y.-P. Sun, J. Am. Chem. Soc., 2011, 133, 4754-4757.

8. H. de Lasa, E. Salaices, J. Mazumder and R. Lucky, Chem. Rev., 2011, 111, 5404-5433.

9. F. H. Isikgor and C. R. Becer, Polym. Chem., 2015, 6, 4497-4559.

10. L. C. Rodriguez and D. O'Connell, Nature, 2011, 476, 283.

11. C. Somerville, H. Youngs, C. Taylor, S. C. Davis and S. P. Long, Science, 2010, $329,790-792$.

12. P. Shen, J. Gao, J. Cong, Z. Liu, C. Li and J. Yao, ChemistrySelect, 2016, 1, 1314-1317.

13. S. Rai, B. K. Singh, P. Bhartiya, A. Singh, H. Kumar, P. K. Dutta and G. K. Mehrotra, J. Lumin., 2017, 190, 492-503.

14. X. Wen, L. Shi, G. Wen, Y. Li, C. Dong, J. Yang and S. Shuang, Sens. Actuators, B, 2015, 221, 769-776.

15. W. Lu, X. Qin, S. Liu, G. Chang, Y. Zhang, Y. Luo, A. M. Asiri, A. O. Al-Youbi and X. Sun, Anal. Chem., 2012, 84, 5351-5357.

16. Y. Liu, Y. Zhao and Y. Zhang, Sens. Actuators, B, 2014, 196, 647-652.

17. X. Qin, W. Lu, A. M. Asiri, A. O. Al-Youbi and X. Sun, Catal. Sci. Technol., 2013, 3, 1027-1035. 
18. G. S. Das, J. P. Shim, A. Bhatnagar, K. M. Tripathi and T. Kim, Sci. Rep., 2019, 9, 15084.

19. H. Wang, J. Zhuang, D. Velado, Z. Wei, H. Matsui and S. Zhou, ACS Appl. Mater. Interfaces, 2015, 7, 27703-27712.

20. W. Meng, X. Bai, B. Wang, Z. Liu, S. Lu and B. Yang, Energy Environ. Mater., 2019, 2, 172-192.

21. A. Tyagi, K. M. Tripathi, N. Singh, S. Choudhary and R. K. Gupta, RSC Adv., 2016, 6, 72423-72432.

22. J. Schneider and D. W. Bahnemann, J. Phys. Chem. Lett., 2013, 4, 3479-3483.

23. Y. Pellegrin and F. Odobel, C. R. Chim., 2017, 20, 283-295.

24. F. Pulizzi and W. Sun, Nat. Nanotechnol., 2018, 13, 633-633.

25. P. J. J. Alvarez, C. K. Chan, M. Elimelech, N. J. Halas and D. Villagrán, Nat. Nanotechnol., 2018, 13, 634-641.

26. A. S. Hainer, J. S. Hodgins, V. Sandre, M. Vallieres, A. E. Lanterna and J. C. Scaiano, ACS Energy Lett., 2018, 3, 542-545.

27. Y. Li, D. Gao, S. Peng, G. Lu and S. Li, Int. J. Hydrogen Energy, 2011, 36, 4291-4297.

28. Y. Li, G. Lu and S. Li, Appl. Catal. A, 2001, 214, 179-185.

29. X. J. Zheng, L. F. Wei, Z. H. Zhang, Q. J. Jiang, Y. J. Wei, B. Xie and M. B. Wei, Int. J. Hydrogen Energy, 2009, 34, 9033-9041.

30. J. Kim, D. Monllor-Satoca and W. Choi, Energy Environ. Sci., 2012, 5, 76477656.

31. B. C. Hodges, E. L. Cates and J.-H. Kim, Nat. Nanotechnol., 2018, 13, 642650. 
32. V. M. Daskalaki, M. Antoniadou, G. Li Puma, D. I. Kondarides and P. Lianos, Environ. Sci. Technol., 2010, 44, 7200-7205.

33. K. Li, Z. Zeng, L. Yan, M. Huo, Y. Guo, S. Luo and X. Luo, Appl. Catal., B, 2016, $187,269-280$.

34. S. Ahmed, M. G. Rasul, R. Brown and M. A. Hashib, J. Environ. Manag., 2011, 92, 311-330.

35. A.-J. Simamora, F.-C. Chang, H. P. Wang, T.-C. Yang, Y.-L. Wei and W.-K. Lin, Int. J. Photoenergy, 2013, 2013, 419182.

36. L. Guo, Z. Yang, K. Marcus, Z. Li, B. Luo, L. Zhou, X. Wang, Y. Du and Y. Yang, Energy Environ. Sci., 2018, 11, 106-114

37. D. Jassby, T. Y. Cath and H. Buisson, Nat. Nanotechnol., 2018, 13, 670-672.

38. E. de Morais Teixeira, A. C. Corrêa, A. Manzoli, F. de Lima Leite, C. R. de Oliveira and L. H. C. Mattoso, Cellulose, 2010, 17, 595-606.

39. Á. Peralbo-Molina and M. D. Luque de Castro, Trends Food Sci. Technol., 2013, 32, 16-24.

40. A. George, T. J. Morgan and R. Kandiyoti, Energy Fuels, 2014, 28, 6918-6927.

41. M. Carrier, A. Loppinet-Serani, D. Denux, J.-M. Lasnier, F. Ham-Pichavant, F. Cansell and C. Aymonier, Biomass Bioenergy, 2011, 35, 298-307.

42. E. Biagini, F. Barontini and L. Tognotti, Ind. Eng. Chem. Res., 2006, 45, 44864493.

43. L. Abaza, A. Taamalli, H. Nsir and M. Zarrouk, Antioxidants, 2015, 4, 682-698.

44. G. Yang, G. Zhang, P. Sheng, F. Sun, W. Xu and D. Zhang, J. Mater. Chem., 2012, 22, 4391-4395.

45. G. Oza, M. Ravichandran, V.-I. Merupo, S. Shinde, A. Mewada, J. T. Ramirez, S. Velumani, M. Sharon and M. Sharon, Sci. Rep., 2016, 6, 21286. 
46. Y. Qin, N. Liu, H. Li, Y. Sun, L. Hu, S. Zhao, D. Han, Y. Liu, Z. Kang and L. Niu, J. Phys. Chem. C, 2017, 121, 27546-27554.

47. Z. Yang, M. Xu, Y. Liu, F. He, F. Gao, Y. Su, H. Wei and Y. Zhang, Nanoscale, 2014, 6, 1890-1895.

48. J. Zhang, Z. Xia and L. Dai, Sci. Adv., 2015, 1, e1500564.

49. A. C. Ferrari and D. M. Basko, Nat. Nanotechnol., 2013, 8, 235-246.

50. Z. Wang, L. Cao, Y. Ding, R. Shi, X. Wang, H. Lu, Z. Liu, F. Xiu, J. Liu and W. Huang, RSC Adv., 2017, 7, 21969-21973.

51. J. Zhou, C. Booker, R. Li, X. Zhou, T.-K. Sham, X. Sun and Z. Ding, J. Am. Chem. Soc., 2007, 129, 744-745.

52. W. Gao, L. B. Alemany, L. Ci and P. M. Ajayan, Nat. Chem., 2009, 1, 403-408.

53. C. A. Caputo, M. A. Gross, V. W. Lau, C. Cavazza, B. V. Lotsch and E. Reisner, Angew. Chem. Int. Ed., 2014, 53, 11538-11542.

54. D. Lu, H. Wang, X. Zhao, K. K. Kondamareddy, J. Ding, C. Li and P. Fang, ACS Sustainable Chem. Eng., 2017, 5, 1436-1445.

55. H. Liu, Z. Jin, Z. Xu, Z. Zhang and D. Ao, RSC Adv., 2015, 5, 97951-97961.

56. Y. Wu, H. Wang, W. Tu, S. Wu, Y. Liu, Y. Z. Tan, H. Luo, X. Yuan and J. W. Chew, Appl. Catal. B, 2018, 229, 181-191.

57. C. H. Choi, L. Lin, S. Gim, S. Lee, H. Kim, X. Wang and W. Choi, ACS Catal., $2018,8,4241-4256$.

58. Y. Wu, H. Wang, W. Tu, Y. Liu, S. Wu, Y. Z. Tan and J. W. Chew, Appl. Catal. $B, 2018,233,58-69$.

59. L. N. Qiao, H. C. Wang, Y. D. Luo, H. M. Xu, J. P. Ding, S. Lan, Y. Shen, Y. H. Lin and C. W. Nan, J. Am. Ceram. Soc., 2018, 101, 3015-3025. 
60. H. Fu, L. Yang, D. Hu, C. Yu, Y. Ling, Y. Xie, S. Li and J. Zhao, Int. J. Hydrog. Energy, 2018, 43, 10359-10367.

61. P. Tian, X. He, W. Li, L. Zhao, W. Fang, H. Chen, F. Zhang, W. Zhang and W. Wang, J. Mater. Sci., 2018, 53, 12016-12029.

62. B. C. M. Martindale, E. Joliat, C. Bachmann, R. Alberto and E. Reisner, Angew. Chem. Int. Ed., 2016, 55, 9402-9406.

63. L. H. Nowell, A. S. Ludtke, D. K. Mueller and J. C. Scott, USGS open-file report 2011, 2011-1271.

64. G. Zhang, L. Lihua, L. Guosheng, Z. Yongfan, S. Aleksandr, Z. Spiros, W. Xinchen and A. Markus, Angew. Chem. Int. Ed., 2018, 57, 9372-9376.

65. K. Brown, River Cam water quality data, British environmental agency, 2017.

66. J. Kim, J. Lee and W. Choi, Chem. Commun., 2008, 0, 756-758.

67. Z. Jiang, W. Wan, W. Wei, K. Chen, H. Li, P. K. Wong and J. Xie, Appl. Catal. $B, 2017,204,283-295$.

68. Y. C. Nie, F. Yu, L. C. Wang, Q. J. Xing, X. Liu, Y. Pei, J. P. Zou, W. L. Dai, Y. Li and S. L. Suib, Appl. Catal. B, 2018, 227, 312-321.

69. A. E. Lanterna and J. C. Scaiano, ACS Energy Lett., 2017, 2, 1909-1910.

70. M. A. Gross, A. Reynal, J. R. Durrant and E. Reisner, J. Am. Chem. Soc., 2014, 136, 356-366. 
ToC artwork and text

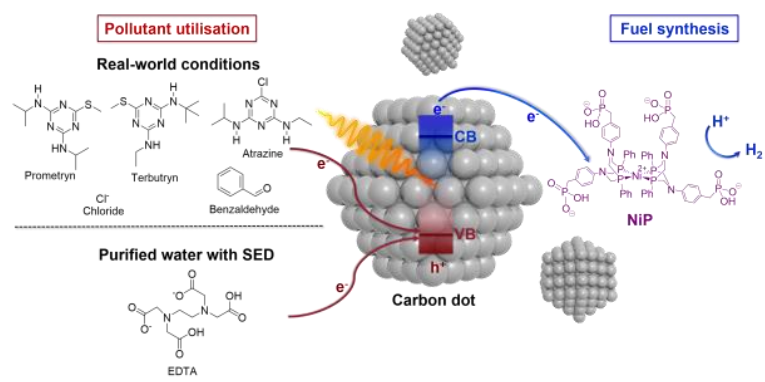

Carbon dots from biomass waste as efficient photoabsorbers for sustainable and scalable coupled solar-driven $\mathrm{H}_{2}$ evolution and pollutant utilisation. 